\title{
Myelodysplastic Syndromes with Monosomy 7 and Granulocyte Colony-Stimulating Factor
}

One of the major concerns in the therapeutic application of granulocyte colony-stimulating factor (G-CSF) is the stimulation of the neoplastic cell growth especially in patients with myelodysplastic syndromes (MDS) and acute leukemias. More recently, the development of MDS with monosomy 7 has been increasingly reported in aplastic anemia (AA) patients undergoing G-CSF therapy (1). Monosomy 7 is frequently seen in childhood MDS (2) and may also occur in chronic neutropenic children other than AA following G-CSF (3). MDS or acute myelogenous leukemia (AML) with monosomy 7 may occur in adult patients with AA following treatment with G-CSF with or without immunosuppressants as reported in this issue (4).

MDS and/or AML are well-known late complications of anti-thymocyte or anti-lymphocyte globulin (ATG or ALG) therapy in severe AA (5-8). The reported incidence of MDS and/or AML following ATG or ALG ranges from 4 to 9\% (58 ). The difference may be due to the differences in the duration of follow-up and the number of patients. An update of the largest series (7) identified 31 cases of MDS or AML in 619 AA patients with at least 6 months of follow-up after ALG. The 10year cumulative risk for MDS or AML has been estimated as $9.6 \%$ and $6.6 \%$, respectively (7). Since these late complications were not found in AA patients following allogeneic bone marrow transplantation (5-8), several hypotheses have been proposed. The late occurrence of these complications suggests that a quantitative chronic stem cell defect by itself could be a risk for the subsequent development of MDS or AML. Given the limited number of stem cells in AA, clonal succession may thus explain the late occurrence of clonal hemopathy. Alternatively, unknown cause(s) could cause more than one abnormality, favoring AA first and MDS thereafter. A third hypothesis is that the evolution of MDS and/or AML can be part of the natural history of marrow aplasia. As is evident from the old term "aplasia/leukemia syndrome", this hypothesis postulates that at least a proportion of AA may be a premalignant clonal disorder, often presenting with preleukemic state. Although it remains unsettled which hypothesis is correct, improved survival has obviously contributed to the apparent increase in the incidence of these late complications.

A 1994 report of a survey of G-CSF treatment in 67 Japanese children (3) with AA recorded 3 cases of fatal MDS and/or AML with monosomy 7. The development of MDS and AML occurred between 23 and 28 months from the start of G-CSF therapy. The cumulative dose of G-CSF in these cases varied widely ( 2.9 to $500 \mathrm{mg}$ ). The actual incidence of MDS and/or AML in AA patients surviving more than 2 years was $14.3 \%$
(3), which may not be significantly high as compared with the estimated rate of MDS and/or AML in AA patients in earlier reports when G-CSF was not available $(7,8)$. It should be noted that evolution of MDS and/or AML from AA included cases with monosomy 7 even prior to the days of cytokine treatment (9). Nonetheless, the association between MDS and/or AML with monosomy 7 and G-CSF therapy appears noteworthy. At present, there is no explanation as to why G-CSF may stimulate clones with 7 monosomy.

A prospective study is warranted to determine the incidence of these late complications and to answer the question of whether the relative frequency of monosomy 7 may be related to G-CSF therapy. In view of the low mitotic rates, all AA patients receiving G-CSF should be examined at regular intervals using a more sensitive technique, such as fluorescent in situ hybridization.

Yataro YoshiDA, MD First Division, Department of Medicine, Kyoto University, Sakyo, Kyoto 606

\section{References}

1) Kojima S, Tsuchida M, Matsuyama T. Myelodysplasia and leukemia after treatment of aplastic anemia with G-CSF. N Engl J Med 326: 1294, 1992.

2) Yoshida Y, Stephenson J, Mufti GL. Myelodysplastic syndrome from morphology to molecular biology. Part I. Classification, natural history and cell biology in myelodysplasia. Int J Hematol 57: 87, 1993.

3) Imashuku S, Hibi S, Nakajima F, et al. A review of 125 cases to determine the risk of myelodysplasia and leukemia in pediatric neutropenic patients after treatment with recombinant human granulocyte colony-stimulating factor. Blood 84: 2380, 1994.

4) Ohsaka A, Sugahara Y, Imai Y, et al. Evolution of severe aplastic anemia into myelodysplasia with monosomy 7 after granulocyte colony-stimulating factor, erythropoietin and high-dose methylprednisolone combination therapy. Internal Medicine 34: 892, 1995.

5) Paquette RL, Tebyani N, Frane M, et al. Long-term outcome of aplastic anemia in adults treated with antithymocyte globulin: Comparison with bone marrow transplantation. Blood 85: 283, 1995.

6) Doney K, Kopecky K, Storb R, et al. Long-term comparison of immunosuppressive therapy with anti-thymocyte globulin to bone marrow transplantation in aplastic anemia. in: Aplastic Anemia and Other Bone Marrow Failure Syndromes, Shahidi NT, Ed. Springer, New York, 1989, p.104.

7) Socie G, Henry-Amar M, Bacigalupo A, et al. Malignant tumors occurring after treatment of aplastic anemia. N Engl J Med 329: 1152, 1993.

8) Speck B, Tichelli A, Gratwohl A, Nissen C. Treatment of severe aplastic anemia: A 12-year follow-up of patients after bone marrow transplanta- 
tion or after therapy with antilymphocyte globulin. in: Aplastic Anemia and Other Bone Marrow Failure Syndromes, Shahidi NT, Ed. Springer, New York, 1990, p.96.
9) de Planque MM, Kluin-Nelemans HC, van Krieken HJM, et al. Evolution of acquired aplastic anemia to myelodysplasia and subsequent leukaemia in adults. Br J Haematol 70: 55, 1988. 